\title{
Seminario de Prospectiva, sostenibilidad y transdisciplina
}

\author{
María BeATriz AbacA ${ }^{1}$
}

El Seminario "Prospectiva, sostenibilidad y transdisciplina" de la Ecocucea fue un excelente "kairos" de reflexiones y diálogo de saberes a partir de los cuales realizó una sencilla reflexión articulando tales saberes con los campos de la filosofía y la pedagogía, en relación con el cuestionamientosobre las grandes problemáticas que entran en juego en este contexto de "colapso socioambiental": lapérdida de biodiversidad y el tiempo en su carácter de "run out of time" necesarios para una re-existencia ecologizada post-pandemia.

\section{Prospectiva superadora de las posibilidades del desarrollo sostenible para la restitución de la biósfera. El tiempo como determinante para la restitución de la biodiversidad integral}

Desde la problemática pandémica por el Covid-19 de origen zoonótica en la cual nos encontramos, esfundamental replantearnos el juego entre prospectiva, restitución de la biodiversidad y el concepto detiempo; podríamos esbozar la problemática planteándonos: ¿Tendremos la posibilidad de una prospectiva a partir de la cual logremos recrear el tiempo para la restitución de la biodiversidad? Parala profundización de la problemática que arroja una enorme preocupación personal, parto de un esbozo atrevido de diálogo de saberes entre algunas temáticas trabajadas en el seminario, el teorema de IlyaPrigogine, Premio Nobel de Química, y Enrique Dussel, creador de la filosofía de la liberación latinoamericana.

Partiendo de Godet y Durance (2009), ${ }^{2}$ los conceptos de prospectiva, estrategia y planificación manifiestan que están íntimamente relacionados, en los cuales cada uno apela a los otros y define a la prospectiva como:

1 Doctoranda en Pedagogía Ecosocial de la universidad de Villa María, Córdoba, Argentina.

2 Goyeneche, Guadalupe. (s/f). Introducción a la prospectiva: Síntesis metodológica. Tomás Parodi, capítulo 1: "Definiciones de la prospectiva y su relación con el pensamiento estratégico de largo plazo". 
"A este fin, se trata de investigar futuros posibles, de explorar posibilidades; pero también que se denominan los futuros probables -y explorar aquellos que dentro de los probablesson los preferibles - y que se conocen como los futuros deseados".

Retomando la fundamental consideración del concepto de tiempo para la prospectiva, si realizamos unparalelo con el teorema de Prigogine podemos articular:

1. Los probables son una restricción del campo de los posibles y cuentan con mayor potencial de realizarse, a partir de su confrontación con los hechos, los datos, las percepciones calificadas de los decisores y los expertos; son los normalmente considerados por la ciencia clásica dentro de un relativismo temporal o el modelo newtoniano más reduccionista aún, a partir del cual se plantea un solo modelo de futuro ${ }^{3}$ según Prigogine.

2. Los futuros posibles, todos aquellos que pueden acaecer, pero que sin embargo podrían se atraídospor detractores y multiversidad de variables, en el caso de los sistemas complejos; tal ejemplo de la mecánica cuántica.

3. Los futuros deseables consultan los valores, las expectativas y las aspiraciones de las personas, remiten al deseo de proyectarse hacia el encuentro del futuro, dentro de un contexto social e históricodeterminado de antemano. El salto al modelo de los futuros deseables se encuentra en sintonía con la "nueva alianza" planteada por Prigogine que consiste en la relación "ciencia y naturaleza". Una prospectiva desde esta perspectiva se resignifica entramándose en el "tiempo creador" de las narrativasposibles, abriendo posibilidades al sistema, multiversidad de probabilidades que a manera de sistemaorgánico, abierto a la energía y la materia, participe de la reversibilidad temporal en cuanto "tiempo en el corazón" de la experiencia.

4. Esta perspectiva de la prospectiva entramada en la narrativa, se abre a la evolución de las leyes fundamentales de la naturaleza, cambiando la clasificación de las ciencias antes duras: Newton, Einstein, Shoundinger, que hablaban de certezas, y las ciencias humanas como la economía, sociología y filosofía que hablaban de posibilidades, en el universo complejo de la incertidumbre, todas remiten a las "posibilidades", convocándonos a "pensar lo incierto", ya la razón no es más un sinónimo de certeza y las probabilidades un sinónimo de ignorancia. Recobra un significado muy importante la filosofía como narrativa superadora del dualismo, del relativismo y lo autómata hacia la construcción en este sentido de la "nueva alianza".

5. Esta "nueva alianza" permite que la prospectiva se transforme y pase de ser un acontecimiento individual a ser global de las poblaciones, como la biología de Darwin ha pasado a ser biología de las poblaciones. Este futuro que no está dado, sino que está haciéndose, introduce la "narrativa" en la evolución de las leyes fundamentales de la naturaleza, destacando el protagonismo de la humanidad

3 https://www.youtube.com/watch?v=tuqrvPQ7nAk. Grandes pensadores del siglo XX. Consultado 19/10/2020.

4 Prigogine, Ilya. (s/f). Fin de las certidumbres. https://www.academia.edu/29714772/Prigogine?email_ work_card=view. Consultado 20/10/2020 Ilya Prigogine, autor belga nacido en 1917, Premio Nobel en 1977 por la introducción del conceptode "estructuras disipativas". 
en la creación de la nueva realidad"; de aquí la reinvención de la filosofía en la filosofíade la liberación, la "ética de la liberación", una narrativa es irreductible a una sola trayectoria ya que hay que considerar las diversas posibilidades y la probabilidad de los sistemas humanos y ambientales abiertos a las novedades, a la innovación de la creatividad que supera el determinismo y nos devela la implicancia "ética".

En este mismo sentido, mirar al futuro transforma el presente, o sea que la previsión invita a la acción. La prospectiva es estratégica; si no lo es por los resultados, lo es por sus intenciones, y la estrategia apela a la prospectiva para aclarar las decisiones que comprometen el futuro (Berger, 1959).

\section{Prospectiva como "ética liberadora" de los territorios de conquista}

En cuanto a la inserción en la realidad cotidiana y la movilización de la inteligencia colectiva, partiendo del aporte de Bourbon-Busset (1959) en cuanto a la importancia del tiempo de dilucidaciónde la prospectiva y a la profundización del sentido general y profundo de los hechos observados para luego planificar las acciones:

Maurice Blandel insistía en que "no se debe prever el futuro, hay que prepararse para él”. Así, reflexionar es un paso previo a la acción. Por lo tanto, para serle útil al hombre de accióny para ser eficaz, la prospectiva debe dilucidar el sentido general y profundo de los hechos observados, elaborar planes y programas, recomendaciones de aplicación inmediata, mostrarideas de acción, fijar objetivos alcanzables.

[...] la forma de "concebir un futuro deseado así como los medios reales para alcanzarlo”.Por su parte, Carlos Matus (1993) concibe la planificación estratégica como un instrumento para gobernar y para actuar; la entiende como una herramienta que se alimenta de un proceso prospectivo de construcción de caminos posibles que permite lidiar con la incerteza, afrontar problemas críticos, negociar con multiplicidad y diversidad de actores, intervenir en el juego social, y conducir y liderar el proceso deplanificación a largo plazo. Estos elementos están en constante retroalimentación y sutransformación dinámica es un desafío para la puesta en marcha de acciones. Así, la prospectiva estratégica es fundamental para construir acuerdos, para generar alianzasorientadas a la acción y para consolidar rumbos estratégicos que permitan ejecutar políticas (Bourbon-Busset, 1959).

Podremos realizar un paralelo en sintonía con el aporte de la Ética de la liberación ${ }^{5}$ de Enrique Dussel y el rol de la filosofía como disciplina de las "posibilidades" que consiste en la defensa de lavida humana en comunidad y la defensa de la biósfera. El gran aporte de sus 14 tesis y de sus tres principios desde y para un horizonte mundial planetario son fundamentales para generar metodologías de territorialización

5 Albán A., Adolfo, y Rosero, José R. (2016). Colonialidad de la naturaleza: ¿Imposición tecnológica y usurpación epistémica? Interculturalidad, desarrollo y re-existencia. Col. Nómadas, núm. 45, octubre, pp. 27-41. Bogotá, Colombia: Universidad Central. Disponible en: http:/www.redalyc.org/articulo. oa? id=105149483004 
geopolítica, hacia la construcción de los cuidados. El principio material a partir del cual se fundamenta la defensa de la vida en comunidad como principio de inclusióny la defensa de la biósfera, tal cual una brújula que nos orienta a la construcción de los principios siguientes a manera de recursividad. El principio formal, partiendo de que la construcción de los cuidados debe partir de las voces de todos y el aprendizaje de la inclusión de todas y todos a manera de metodología que se hace experiencia territorial para la recursividad en la construcción del pensamiento liberador y latinoamericano. La construcción del consenso compartido mediante debate,manifestando que todos tienen una voz y el alcance del consenso que le da la validez formal mediante los argumentos racionales, sin violencia con el objetivo del avance en el cuidado y restitución de la biósfera con sus diversidades y dentro de ella, la vida humana y su derecho a la vida comunitaria. Porúltimo, el principio de factibilidad la construcción de los consensos debe tender a la factibilidad de poder construirse. Ello implica que las discusiones y aportes a la construcción de la ética de la liberación no queden en constructos teóricos sino más bien sean productos de una praxis territorial esdecir aplicables en campos prácticos de manera que el mundo haya más justicia, más paz y restitucióndel medio ambiente. Principios fundamentales a partir de los cuales se concretiza la corresponsabilidadpor el "Otro" superando la negación del ser humano y de la naturaleza como objetos del sistema capitalista y la colonialidad del poder. La afirmación de la vida en comunidad que es también la biósfera en la construcción del proyecto de liberación.

Esta dimensión de la ética liberadora es la novedad del tiempo creador, que destaca la superación deldeterminismo y las certezas del rol creador, responsable de la humanidad, entramado con el tiempo desde la perspectiva de las posibilidades.

\section{Del paradigma de la moral científica al paradigma de la ética trans-sistémica o ciencia digna}

El aporte de Prigogine en cuanto al protagonismo de la humanidad en la participación "ética" en la construcción de un "tiempo creativo", para un mundo en construcción ampliando el concepto de las leyes de la naturaleza en el cual se incluyen la irreversibilidad de los sistemas cerrados y la reversibilidad de los sistemas abiertos, logrando la posibilidad de construcción de la nueva alianza dela ciencia con la naturaleza.

Las relaciones de las problemáticas territoriales socioambientales, como "estructuras disipatorias"según la teoría de Prigogine, son de la tercera realidad originada a partir del sistema abierto con una alta entropía, sujeto a una gran posibilidad aleatoria de clinamen. ${ }^{6}$

6 Término latino acuñado por Lucrecio (Titus Lucrecius Carus), seguidor de la filosofía de Epicuro, para traducir el griego parénklesis con el que Epicuro se refería a la desviación espontánea de la trayectoria rectilínea que experimentaban los átomos paraexplicar su agregación con otros átomos. El clinamen es, pues, la espontánea desviación de la trayectoria de los átomos, que rompe la cadena causal, determinista, de su movimiento, introduciendo así un fundamento físico para justificar la 
En resumen, "una ciencia disruptiva" del doctor Andrés Carrasco o el concepto de "ciencia digna", del quehabla el doctor Damián Verseñazzi, de la Facultad de Medicina de Rosario, podemos ver el momento dela "ética en sentido trans-sistémico", ya que los científicos realizan la opción de ponerse en el lugar de las víctimas, en este caso del glifosato y en función epistémica, ya que en su criticidad cuestionan elsentido de la ciencia misma, remitiendo a la pregunta: ¿ciencia para qué? ¿Ciencia para quiénes?, sosteniendo la valoración absoluta de la vida humana, superando la moral del sistema. Hoy el espacio de salud socioambiental como de la UNR, liderado por Verseñazzi, se manifiesta como experiencia intercultural abierta al diálogo de saberes. Considero muy valorable este espacio ya quemanifiesta una experiencia de territorio, epistémica y de descolonización de la naturaleza colonizaday que convoca desde su praxis territorial a la construcción de la "ética en sentido trans-sistémico" en forma continua. La problemática ambiental que desarrolla Albán y Rosero devela de la misma manera la raíz ontológica de la problemática en cuanto al concepto de naturaleza, su diversidad y alconcepto de hombre separado, escindido en relación con la naturaleza y con ello el alejamiento de la mirada del "nosotros" y la raíz colonizadora eurocéntrica del falso concepto de desarrollo que imprimióel capitalismo, generando la monocultura del consumo mediante la explotación y el extractivismo despiadados de biodiversidad, que es diversidad biológica y diversidad cultural.

\section{De las competencias a la construcción comunitaria de saberes complejos. ¿Qué enseñar y aprender, para qué, para quiénes y cómo?}

Tina Evans, en sintonía con Prigogine coincide con la perspectiva superadora de la realidad de la físicaclásica, como la mecánica cuántica y se adhiere al concepto de conjuntos interconectados, sistemas abiertos, como organismos vivos:

[...] reconozcamos lo que muchas culturas tradicionales e indígenas han reconocido durante milenios: que lo social está incrustado en lo ecológico y que nosotros, como individuos y comunidades, somos sólo partes de conjuntos profundamente interconectados.

Del teorema de Prigogine sobre las "estructuras disipativas" se desprende la idea de irreversibilidad de la entropía planteada por la segunda ley de la termodinámica, la concepcióndel mundo en evolución que se va realizando con aumento de entropía, por lo cual indica una realidadde desequilibrio y caos.

Ciertamente, ésta es una concepción del mundo en evolución. Pero entonces, ¿cómo encaja en la descripción fundamentalmente atemporal que proporcionan "las

acción libre, en los seres humanos, y el azar. https://www.webdianoia.com/glosario/display. Consultado 28/10/20.

7 https://www.youtube.com/watch?v=u9BSb8fDgTADr. Andrés Carrasco. Última intervención pública de un indispensable que se nosfue. 
leyes de la naturaleza? Prigogine destaca un reciente informe de C. K. Biebricher, G. Nicolis y P. Schuster, quienes escriben:

El mantenimiento de la organización de la naturaleza no se consigue —ni se puede conseguir - con una dirección central; el orden sólo se puede mantener mediante la autoorganización. Los sistemas autoorganizativos. Permitir las condiciones externas. Hay que destacar la superioridad de los sistemas autoorganizativos sobre la tecnología humana convencional [...]. Un ejemplo que ilustra esta superioridad son los sistemas biológicos en los que se pueden formar productos complejos con el máximo de precisión, eficacia y rapidez. 8

Esta diferenciación que plantea destacando la superioridad de los sistemas "autoorganizativos", tomando como ejemplo los biológicos para los cuales sólo es necesario crear un mínimo de condiciones externas, desde las microestructuras a las macroestructuras, y en la medida que las estructuras disipativas aumentan su orden interno, Prigogine afirma repetidamente, a diferencia de laspredicciones de la termodinámica tradicional, que el sistema autoorganizado disminuye su entropía. ${ }^{9}$

Nos focalizaremos en una de las competencias educativas planteadas por Tina Evans:

"La sostenibilidad va más allá de la crítica y la comprensión para crear e implementar estrategias para el cambio personal, social y organizacional; requiere competencia creativa y estratégica."

Esta competencia creativa participa de la acción restitutiva de la biodiversidad ambiental, tal cual sistema biológico, abierto de intercambio de materia y energía con capacidad de autoorganización delos diferentes territorios rurales y urbanos.

\section{La Universidad de Guadalajara como sistema biológico, autoorganizado para la reconstitución de la biodiversidad entramada en el tiempo de la creación}

La universidad como espacio urbano no es estructura edilicia, sino oportunidad para convertirse en sistema abierto biológico con fronteras permeables y flexibles con sus interacciones, redes, flujos y dinámicas. Es portadora de espacios propios, comunes en el marco de la justicia ambiental de acceso a los beneficios de ésta y a sus recursos: suelo, agua, calidad ambiental. La universidad como sistemasocial, ecológico, económico y político en su territorio definido por un patrón poblacional con una administración y actividades de formación pedagógica y de construcción de subjetividades. Es una convocatoria al desafío de la construcción comunitaria de saberes vinculados a la alfabetización ecológica integral de manera que la riqueza del diálogo de saberes pueda construirse mediante la permeabilidad de las fronteras académicas con los territorios. La construcción de una universidad para todas y todas.

8 https://www.academia.edu/29714772/Prigogine. Recuperado 20/10/20.

9 http://filoexactas.exactas.uba.ar/olimpia/papers/prigoginebiologia.pdf 
A continuación realizaré el esbozo de algunas líneas a manera de propuesta de acción transformadora socioambiental con el objetivo de "empoderar a la Universidad como centrode alfabetización ecológica integral".

- Construcción de una "Universidad abierta a las comunidades y a los territorios", permear las fronteras (construcción de una ciencia digna).

- Empoderar a la Universidad como centro cultural socioambiental donde todas y todos puedanacceder al diálogo de saberes (conocimiento como bien común mundial).

- Fundar una "cátedra abierta socioambiental" de carácter transdisciplinar como acción estratégica de microsistema que se despliegue recursivamente a los diversos espacios yestamentos universitarios.

a. Cátedra abierta como espacio de educación formal y transdisciplinar mediante

la elaboraciónde propuestas socioambientales entramadas en el currículum universitario.

b. Cátedra abierta como espacio de educación popular abierto a la comunidad.

- Conformación del "Centro de Estudiantes Socioambiental” y su participación en el Consejo Socioambiental comunitario.

- Impulsar la transformación curricular, transdisciplinar y sistémica como modelos pedagógicos de apertura a las comunidades y territorios con la modalidad del aprendizaje situado y de servicio socioambiental.

- Elaboración de una propuesta "proyecto fin de carrera" para los estudiantes en sus últimos añoscon acción de voluntariado socioambiental en diversos territorios.

- Propuesta de campamento estudiantil "fin de carrera" con el objetivo de brindar servicio a lascomunidades.

- Experimentar como Centro Universitario la construcción comunitaria de la trasformación del intersticio rural y urbano. Iniciar la construcción de las ciudades del futuro desde el corazón universitario: "Universidades verdes", resignificando el uso de los espacios y el suelo, jardines, techos, convirtiéndose en una metodología innovadora, que puede contribuir de manera significativa a la regulación del entorno urbano directo, comprendiendo el funcionamiento metabólico de esa ciudad, reducir sus demandas energéticas y mejorar su "flujo metabólico"; será fundamental tener en cuenta:

- Promover proyectos curriculares transversales con las comunidades para la promociónde:

- Sistemas de recuperación de predios, racionalizar el uso del agua. Fomentar campañas de educación ambiental. Crear sistemas de incentivos. La cuestión energética: la utilización eficiente y el ahorro energético.

- Tratamiento de los residuos y elaboración de compostaje: la materia orgánica debe volver a la tierra para evitar su progresivo empobrecimiento y el uso de abonos artificiales.

- Promover nuevos sistemas de producción de alimentos: implementación de cultivos agroecológicos como sistema restituyente. 
- Promover el consumo responsable: en particular de los alimentos. Impulsar los intercambios entre consumidores y productores, facilitar canales alternativos de consumo, disminuir las distancias de intercambio: fomentar por todos los medios posibles el acercamiento de las distancias y los desplazamientos. Impulsar la existencia de huertas y predios productivos dentrode la ciudad. La creación de parques comestibles no sólo recupera servicios ambientales sinotambién las relaciones entre el que produce y cómo lo hace, y el que consume.

- Promover el derecho por la tierra. Denunciar los procesos de especulación inmobiliaria.

- Promover la participación ciudadana en temas ambientales y sociales. ${ }^{10}$

10 Agroecología, Ambiente y Salud: Escudos Verdes Productivos y Pueblos Sustentables Walter A.

Pengue y Andrea F. Rodríguez Editores. ENTIDADES GEPAMA. Buenos Aires y Santiago 2018. 